\title{
Management of an Accidental Ingestion of a Manual Screwdriver in Implant Dentistry: A Case Report
}

\author{
${ }^{1}$ Department of Endodontology, Academic Centre for Dentistry \\ Amsterdam (ACTA), Amsterdam, The Netherlands \\ ${ }^{2}$ Department of Surgical Sciences, University of Cagliari, Cagliari, \\ Italy \\ ${ }^{3}$ Digestive Endoscopy Service, AO Brotzu Hospital, Cagliari, Italy \\ ${ }^{4}$ Private Practice, Cagliari, Italy \\ Eur J Gen Dent 2021;10:60-63.
}

Davide Musu ${ }^{1}$ Antonello Mameli ${ }^{2}$ Pierpaolo Carreras ${ }^{3}$ Gian Nicola Boero ${ }^{4}$

\begin{abstract}
Keywords

- case report

- ingestion

- implant screwdriver

- adenoma

- implants

Ingestion of foreign bodies in dentistry represents an uncommon but possible event and can be life-threatening. An 82-year-old patient presented at our attention for an implant-supported rehabilitation of the maxillary arch. During the placement of the fixed prosthesis, the manual screwdriver was swallowed by the patient. From the moment of the accident, the patient did not develop any symptoms and was monitored for the following days. The instrument was not recovered within 3 days, thus a series of consecutive abdominal $\mathrm{X}$-rays were performed revealing that the screwdriver was located at the level of ileocecal valve with no signs of progression. The screwdriver was removed with colonoscopy under sedation. During this treatment, a polyp was discovered and excised revealing a tubulovillous adenoma depicting low-grade dysplasia. To prevent such accidents, screwdrivers in implant dentistry should be secured with ligatures, or replaced with longer torque control wrenches.
\end{abstract}

\author{
Address for correspondence Antonello Mameli, DDS, Department \\ of Surgical Sciences, University of Cagliari, Cagliari 09100, Italy \\ (e-mail: amamelidds@gmail.com).
}

\section{Introduction}

A possible complications during dental treatment is the ingestion of dental instruments. ${ }^{1}$ This is possible due to different factors such as supine position of the patient, saliva production, age (children less than 5 years old and elderly people), psychological and neurological diseases, ${ }^{2-4}$ wide oropharynx, and absence of gag reflex. ${ }^{1}$ Ingested dental instruments vary in size and shape and can include burs, root pieces, teeth, brackets, endodontic instruments, and implant components. ${ }^{5}$ Usually, swallowed foreign bodies pass through the gastrointestinal system asymptomatically and are expelled via stool in 2 to 4 weeks. ${ }^{1}$ The transit time increases with long and irregularly shaped foreign bodies with a safe passage rate of 63 to $80 \%$ for objects with $<3 \mathrm{~cm}$ of length. ${ }^{6}$ Although rare, complications may appear and in the $75 \%$ are located either at the ileocecal valve or at the rectosigmoid junction. ${ }^{7.8}$

DOI https://doi.org/ $10.1055 / \mathrm{s}-0041-1732774$ ISSN 2320-4753
In these cases, the aid of gastroenterologist is mandatory, and a surgical intervention is needed. Endoscopy retrieval of foreign bodies has a success rate of 94 to $98 \%$; however, complications may happen. ${ }^{10}$ The aim of this article is to report a case of a manual implant screwdriver ingestion and to offer support on the management of such cases.

\section{Case Report}

Our patient, an 82-year-old Caucasian male, came to visit with a chief complaint of masticatory problems due to the absence of numerous teeth and asked for a complete rehabilitation of its oral cavity. The patient had a previous medical history of benign prostatic hyperplasia, hypertensive cardiopathy, and had a pacemaker implanted. He refereed he had a history of tobacco smoking but ceased tobacco use years

(c) 2021. European Journal of General Dentistry.

This is an open access article published by Thieme under the terms of the Creative Commons Attribution-NonDerivative-NonCommercial-License, permitting copying and reproduction so long as the original work is given appropriate credit. Contents may not be used for commercial purposes, or adapted, remixed, transformed or built upon. (https://creativecommons.org/licenses/by-nc-nd/4.0/).

Thieme Medical and Scientific Publishers Pvt. Ltd. A-12, 2nd Floor, Sector 2, Noida-201301 UP, India 
prior to the current entry. He did not consume alcohol. At the time of our examination the patient was taking budesonide $160 \mu \mathrm{g}$ and formoterol fumarate dihydrate $4.5 \mu \mathrm{g}$ by dry powder inhalation (daily) and acetylsalicylic acid (100 mg daily). In December 2019, the patient received an "all-on-four" intervention involving the maxillary arch and the following day the immediate loading of the reinforced acrylic prosthesis was performed. During this occasion the prosthesis was tightened with a torque of $10 \mathrm{~N}$ at the multiunit abutments using a torque controller (Anthogyr, Sallanches, France) and the accesses temporized with Teflon and Cavit (3M ESPE, St. Paul, Minnesota, United States) (-Fig. 1). No complication occurred. The patient was reviewed after 7 days for suture removal. While tightening one of the screws of the upper distal abutments with a manual screwdriver (Nobel Biocare, Zurich, Switzerland), concomitantly with a sudden movement of the patient, the instrument slipped away and was accidentally ingested. The patient was in a supine position and had the perception of swallowing a foreign body. He did not refer any symptom except for cough. The patient was reassured and, according to his family doctor, it was decided to monitor his condition. After 3 days, the patient was still asymptomatic and reported a regular intestinal transit. However, the expulsion of the screwdriver could not be confirmed. Therefore, the patient was referred to a radiological center to verify the presence of the screwdriver in the gastrointestinal tract. An abdominal X-ray was performed, and the instrument was detected and located at the level of the ileocecal valve. The patient was placed under a fiber-rich diet and laxatives (macrogol $13.8 \mathrm{~g}$ per day) to facilitate the elimination of the screwdriver. However, two subsequent abdominal X-rays were repeated after 3 and 6 days which confirmed the screwdriver was still there, thus an endoscopic
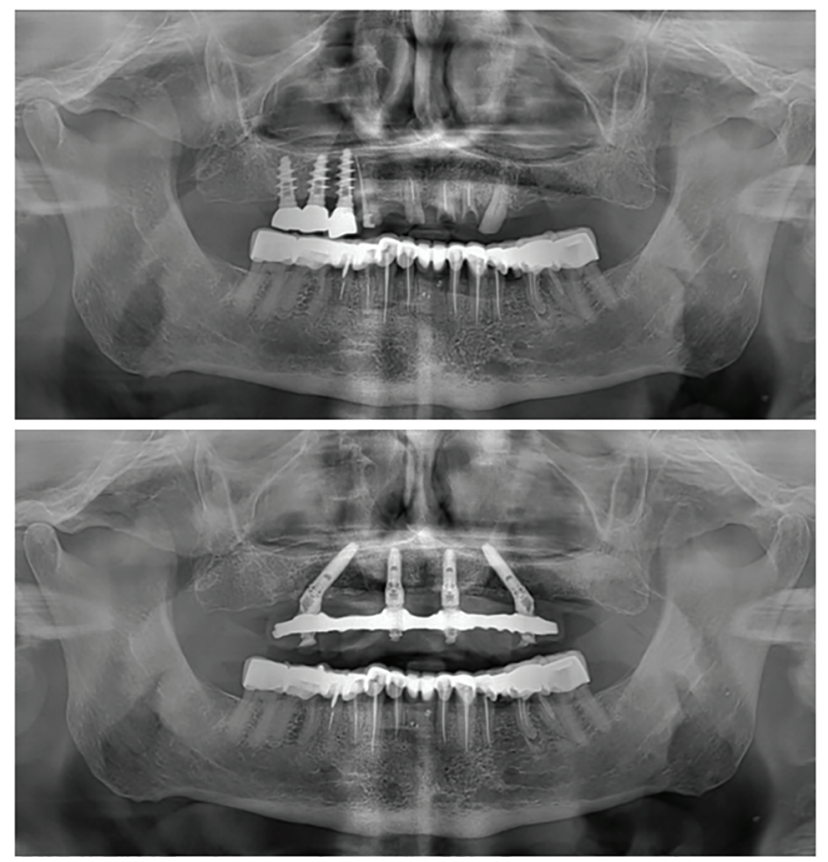

Fig. 1 Orthopantomography of the patient before and after the "all-on-four" intervention. intervention was planned ( - Fig. 2). The patient underwent another preoperative X-ray after which he was hospitalized. Under intravenous sedation full colonoscopy was performed and a $0.8 \times 2.8 \mathrm{~cm}$ titanium screwdriver was located and removed ( - Fig. 3 ). In addition, during the procedure a polyp of the cecum was discovered and excised. Histopathological examination revealed a tubulovillous adenoma showing a low-grade epithelial dysplasia (-Fig. 4). The patient was kept under surveillance at the hospital for the following day and the postoperative recovery was uneventful.

\section{Discussion}

The ingestion and aspiration of foreign bodies during routine clinical dentistry is well documented and it constitutes a rare iatrogenic complication. ${ }^{11}$ Aspiration is less prevalent than ingestion and it is considered a medical emergency since there could be obstruction of the airways and death. Even if the airways are not compromised, the long-term retention may lead to death. ${ }^{2,11}$ As for ingestion, swallowed sharpened objects can potentially cause dramatic complications such as a digestive tract perforation and thus secondary damages to the internal organs which can be lethal for the patient. ${ }^{12}$

In some dental specialties, it is possible to reduce the risk of inhalation or ingestion of dental instruments by isolating the operative field with a rubber dam, nevertheless a case of dental clamp ingestion was reported. ${ }^{13}$ Moreover, in many dental interventions the use of rubber dam is not feasible thus making this inconvenience more susceptible to occur (e.g., implant dentistry). ${ }^{5}$

Indeed, implantology is a specialty at a high risk of ingestion and aspiration since numerous components of small dimensions are used in each procedure whose surfaces become slippery once moistened with saliva. ${ }^{2}$

Different strategies can be embraced to avoid this inconvenience such as packing the oral cavity and the access to the oropharynx with a large piece of gauze. ${ }^{14}$ However, this technique is more suitable in cases of oral interventions performed under sedation, while on an ordinary setting it is less reliable due to the movements of the tongue and in patients with high gag reflexes. Another strategy is to work with the patient on a sitting position. ${ }^{1}$ While this suggestion can be easily applied when working on the mandibular arch it becomes more difficult when the treatment is to be performed in the maxilla since supine position guarantees better ergonomics. The most effective safety measure according to the literature consist of tethering the manual instruments with floss, sutures, or other ligatures, ${ }^{7,11}$ and it is interesting to note that some screwdrivers are fabricated with small holes in the handle for this purpose ( $\mathbf{- F i g}$. 5A). Another alternative that has not been suggested before by scientific literature is the possibility to use longer torque control wrenches with the head of the instrument working intraorally and the handle positioned extraorally ( - Fig. 5B).

There is not a general consensus for the managing of ingestion of foreign bodies during dental treatments. ${ }^{15}$ In the present case, since the patient was calm, asymptomatic, and certain about the ingestion of the screwdriver and 

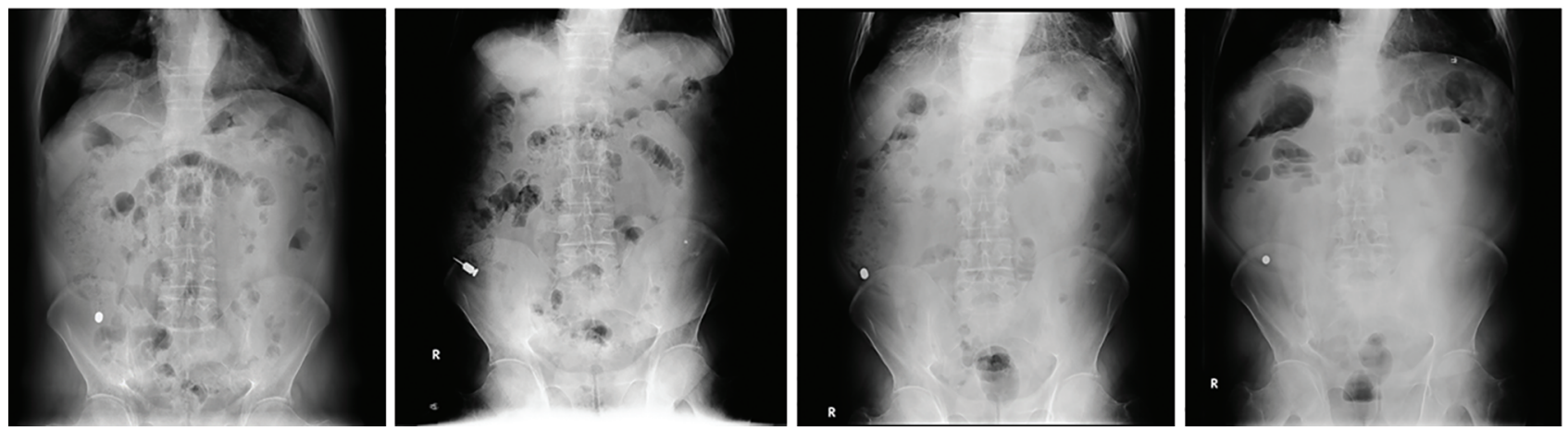

Fig. 2 The abdominal X-rays showing the screwdriver located at the right iliac fossa.
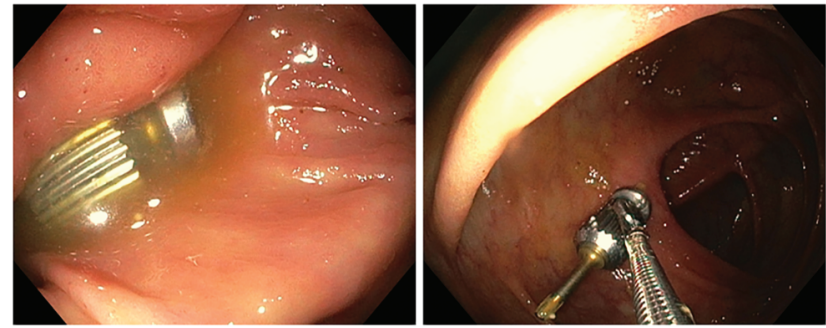

Fig. 3 Endoscopic retrieval of the screwdriver.

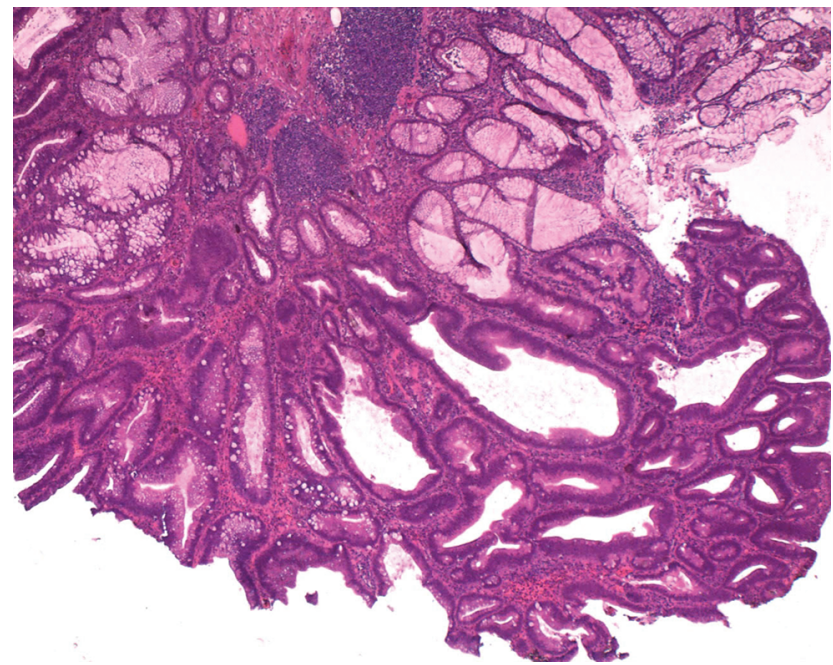

Fig. 4 Hematoxylin and eosin stain $(2 \times)$ showing a tubulovillous adenoma with low-grade dysplasia.

A

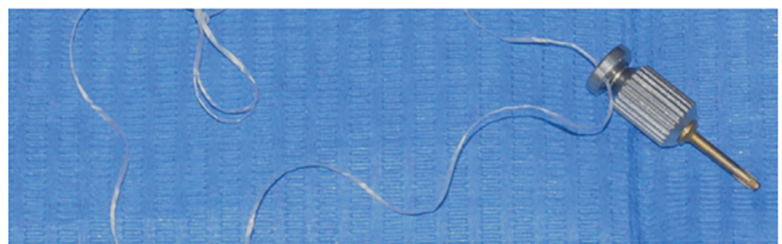

B

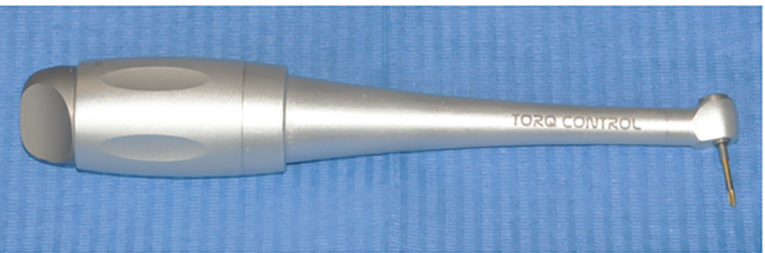

Fig. 5 (A) Manual screwdriver secured with floss. (B) Torque control wrenches. considering the shape and dimension of the object according to the literature examined it was decided for a close monitoring of clinical signs and symptoms and to wait for the expulsion trough the feces. The patient demonstrated to be extremely compliant but since the instrument was not recovered after 3 days a radiographic examination was performed to diagnose the absence of the object or otherwise to monitor the progression of the screwdriver through the gastrointestinal system. This assessment is of paramount importance to avoid an unnecessary endoscopic or surgical intervention. ${ }^{1}$ However, in the present case a diagnosis of retention was made, and the endoscopic intervention was performed.

An interesting feature of the present case is the incidental finding of a polyp that was removed contextually with the procedure which revealed an adenoma. Adenomas are identified in 7 to $41 \%$ of colonoscopies and 2 to $5 \%$ of the polyps that are removed endoscopically contain a malignancy. ${ }^{16}$ Adenomatous polyps are precursors of adenocarcinomas and the higher the degree of cellular dysplasia the higher is the risk of a polyp harboring a colorectal malignancy. In the case of adenomas with low-grade dysplasia, complete endoscopic excision of these lesions is curative. ${ }^{17}$

The dental professional accompanied the patient during all the nondental phases of this report, from the radiological assessment to the pre- and postoperative of the endoscopy. From the patient's perspective this aspect was considered of great importance, and even after the incident, the doctorpatient relationship was not compromised, and the patient is still attending the periodical recall visits.

\section{Conflict of Interest}

None declared.

\section{References}

1 Jain A, Baliga SD. Accidental implant screwdriver ingestion: a rare complication during implant placement. J Dent (Tehran 2014;11(6):711-714

2 Deliberador TM, Marengo G, Scaratti R, Giovanini AF Zielak JC, Baratto Filho F. Accidental aspiration in a patient with Parkinson's disease during implant-supported prosthesis construction: a case report. Spec Care Dentist 2011;31(5):156-161

3 Ireland AJ. Management of inhaled and swallowed foreign bodies. Dent Update 2005;32(2):83-86, 89

4 Worthington P. Ingested foreign body associated with oral implant treatment: report of a case. Int J Oral Maxillofac Implants 1996;11(5):679-681 
5 Tiwana KK, Morton T, Tiwana PS. Aspiration and ingestion in dental practice: a 10-year institutional review. J Am Dent Assoc 2004;135(9):1287-1291

6 Lee JH, Lee JS, Kim MJ, Choe YH. Initial location determines spontaneous passage of foreign bodies from the gastrointestinal tract in children. Pediatr Emerg Care 2011;27(4):284-289

7 de Souza JG, Schuldt Filho G, Pereira Neto AR, Lyra HF Jr, Bianchini MA, Cardoso AC. Accident in implant dentistry: involuntary screwdriver ingestion during surgical procedure. A clinical report. J Prosthodont 2012;21(3):191-193

8 Nash PA, Cregan PC. Perforation of the gastrointestinal tract by a toothpick. Med J Aust 1987;147(8):415-416

9 LiZS, SunZX,Zou DW, Xu GM, Wu RP, LiaoZ. Endoscopic management of foreign bodies in the upper-GI tract: experience with 1088 cases in China. Gastrointest Endosc 2006;64(4):485-492

10 Webb WA, McDaniel L, Jones L. Foreign bodies of the upper gastrointestinal tract: current management. South Med J 1984;77(9):1083-1086

11 Yadav RK, Yadav HK, Chandra A, Yadav S, Verma P, Shakya VK. Accidental aspiration/ingestion of foreign bodies in dentistry: a clinical and legal perspective. Natl J Maxillofac Surg 2015;6(2):144-151

12 Yang Z, Wu D, Xiong D, Li Y. Gastrointestinal perforation secondary to accidental ingestion of toothpicks: a series case report. Medicine (Baltimore 2017;96(50):e9066

13 Mejia JL, Donado JE, Posada A. Accidental swallowing of a dental clamp. J Endod 1996;22(11):619-620

14 Greenstein G, Cavallaro J, Romanos G, Tarnow D. Clinical recommendations for avoiding and managing surgical complications associated with implant dentistry: a review. J Periodontol 2008;79(8):1317-1329

15 Pull Ter Gunne L, Wismeijer D. Accidental ingestion of an untethered instrument during implant surgery. Int J Prosthodont 2014;27(3):277-278

16 Markowitz AJ, Winawer SJ. Management of colorectal polyps. CA Cancer J Clin 1997;47(2):93-112

17 Hall JF. Management of malignant adenomas. Clin Colon Rectal Surg 2015;28(4):215-219 